\title{
Pupillometry for Diagnosing Nonconvulsive Status Epilepticus and Assessing Treatment Response?
}

\author{
Sara Hocker
}

๑ 2021 Springer Science+Business Media, LLC, part of Springer Nature and Neurocritical Care Society

Pupillometry has been widely adopted as a tool to monitor patients with altered levels of consciousness in myriad disease states. Research suggests use of pupillometry in identifying acute changes, assessing severity of disease, and assessing treatment effect, and has predominantly been performed in patients with anoxic ischemic brain injury, aneurysmal subarachnoid hemorrhage, and traumatic brain injury [1]. No previous study has explored the potential clinical use of pupillometry in patients with nonconvulsive status epilepticus (NCSE). In "Automated Pupillometry for Assessment of Treatment Success in Nonconvulsive Status Epilepticus," Godau et al. [2] build on prior work in which they documented both a significant reduction in neurological pupil index (NPi) and a greater difference between left and right NPi in confirmed NCSE [3], this time demonstrating (1) a lower NPi in patients with NCSE and (2) association of both abnormal baseline NPi and normalization of miniNPi with treatment response.

This is a single center prospective observational study of 196 NCSE episodes at one medical center, characterizing the pupillary reactivity patterns assessed by use of automated pupillometer. NCSE was defined as altered mental status with confirmatory electroencephalography (EEG). Patients were excluded if treatment was initiated prior to EEG, bilateral pupillometry could not be performed, or if they had experienced a cardiac arrest. There were 61 patients and 68 consecutive NCSE episodes included in the analysis. Patients were treated according

${ }^{*}$ Correspondence: hocker.sara@mayo.edu

Mayo Clinic Minnesota, 200 First Street SW, Rochester, MN 55905, USA

This article is related to the original work available at https://doi.org/10. 1007/s12028-021-01273-6

\section{Springer}

to local standards with $1-2 \mathrm{mg}$ clonazepam, $40 \mathrm{mg} / \mathrm{kg}$ levetiracetam 40 , and $5 \mathrm{mg} / \mathrm{kg}$ lacosamide in stepwise fashion. The authors performed automated pupillometry before and after each treatment step and assessed response or nonresponse to each medication defined as termination of NCSE. They found that the NPi was low in patients with NCSE prior to AED treatment but then increased with NCSE termination. They also performed statistics examining NPi in relation to refractory NCSE. They found an association between favorable treatment response and improvement of pupillary reactivity.

Data interpretation was performed on a single patient basis in a pre-post design. Treatment was standardized initially but individualized in the refractory stage of NCSE. The observational period for most patients was noticeably short $(1 \mathrm{~h})$. The authors controlled for potential confounding factors, including ambient lighting, and disorders that could render data interpretation possible, such as ocular comorbidity, HIE, and prior medication for the NCSE episode. Correction for dosing of antiseizure drug doses was not performed because the anticipated direct drug effect on pupillary function would be to reduce NPi rather than to increase it, which runs counter to the hypothesis of the study. Additionally, nonresponders to clonazepam received higher drug doses than did responders, without major effects on NPi. The preceding factors limited the systematic bias caused by potential individual confounders.

The study suggests that NPi as assessed by pupillometry could be used as a surrogate for NCSE when EEG is not available, adding an objective tool to facilitate rapid bedside diagnosis of NCSE in coma of unknown etiology or after resolution of the convulsive phase of convulsive status epilepticus (to be confirmed by the gold standard EEG), but only after validation and very good 
confirmatory studies. Further, reduced miniNPi may possibly promote early antiseizure treatment, even in the absence of EEG confirmation, which may contribute to a reduction in the incidence of refractory status epilepticus. Unfortunately, the informative value of automated pupillometry seems to decline with longer NCSE duration and during treatment course, which may limit its diagnostic and treatment monitoring value. This is an innovative idea that could ultimately prove a cost-effective way to improve the care (and possibly outcomes) of this population.

\section{Source of support}

No funding was provided for this work.

\section{Conflicts of interest}

I have adhered to ethical guidelines and have no conflicts of interest.

\section{Publisher's Note}

Springer Nature remains neutral with regard to jurisdictional claims in published maps and institutional affiliations.

Received: 5 May 2021 Accepted: 11 May 2021

Published online: 30 July 2021

\section{References}

1. Lussier BL, Olson DM, Aiyagari V. Automated pupillometry in neurocritical care: research and practice. Curr Neurol Neurosci Rep. 2019;19(10):71.

2. Godau J, Bharad K, et al. Automated pupillometry for assessment of treatment success in nonconvulsive status epilepticus. Neurocrit Care. 2021. https://doi.org/10.1007/s12028-021-01273-6.

3. Godau J, Bierwirth C, Rösche J, Bösel J. Quantitative infrared pupillometry in nonconvulsive status epilepticus. Neurocrit Care. 2020. https://doi.org/ 10.1007/s12028-020-01149-1.

4. Mullaguri N, Katyal N, Sarwal A, Beary JM, George P, Karthikeyan N, et al. Pitfall in pupillometry: exaggerated ciliospinal reflex in a patient in barbiturate coma mimicking a nonreactive pupil. Cureus. 2017;9(12):e2004 